\title{
Genre et classification automatique en TAL : le cas de genres journalistiques
}

Genre and NLP : the case of the automatic classification

\section{Amalia Todirascu}

\section{(2) OpenEdition}

12 Journals

Édition électronique

URL : https://journals.openedition.org/linx/3183

DOI : 10.4000/linx.3183

ISSN : 2118-9692

Éditeur

Presses universitaires de Paris Nanterre

\section{Référence électronique}

Amalia Todirascu, "Genre et classification automatique en TAL : le cas de genres journalistiques », Linx [En ligne], 78 | 2019, document 7, mis en ligne le 30 juin 2019, consulté le 28 juillet 2022. URL: http://journals.openedition.org/linx/3183; DOI : https://doi.org/10.4000/linx.3183

Ce document a été généré automatiquement le 28 juillet 2022

Tous droits réservés 


\section{Genre et classification automatique en TAL : le cas de genres journalistiques}

Genre and NLP : the case of the automatic classification

Amalia Todirascu

\section{Introduction}

1 Dans le domaine du Traitement automatique des langues (TAL), plusieurs définitions différentes de la notion du genre sont utilisées selon l'application qui fait appel à cette tâche de classification. Ainsi, on considère que le genre regroupe les documents ayant le même but communicatif, pour un public spécifique (dyslexiques) dans le cadre d'une application de simplification automatique de textes (Gala et al., 2018). Les applications d'extraction terminologique (Todiraşcu et al., 2012) ou de recherche d'information (Petrenz et Webber, 2011) définissent le genre par ses caractéristiques stylistiques communes (méta-langage scientifique, vocabulaire contrôlé, vocabulaire générale ou spécialisé). C'est une perspective étroite qui dépend de l'application visée.

2 En linguistique textuelle, le genre s'inscrit dans une tradition discursive historique (Koch, 1997), ayant un ancrage social et un caractère prescriptif et normatif. Selon Rastier et al. (1994: 222), " un genre est un programme de prescriptions positives ou négatives, et de licences qui règlent aussi bien la génération d'un texte que son interprétation", suivant les normes sociales. Bouquet (2004) appelle genre un «constituant générique du sens» permettant de prendre en compte le contexte extralinguistique dans lequel le texte est interprété. Halliday (1978) considère les genres du point de vue fonctionnel, des indices linguistiques et structurels spécifiques soutiennent le but communicationnel. Ainsi, nous retenons l'aspect normatif et fonctionnel qui conditionne l'expression linguistique (fréquence de certains groupes nominaux, préférence pour des sujets pronominaux ou pour le passif) et le contenu du texte (Rastier, 1989, Bouquet, 2004), mais aussi la dimension extra-linguistique sociale 
de la notion de genre. Ces indices seront utilisés pour la classification automatique des genres discursifs dans le cadre d'une application de classification de genres journalistiques pour la détection de néologismes, dans le cadre du projet Logoscope.

Nous considérons que le genre discursif est défini par la situation de production et de réception du texte (Biber et Conrad, 2009, Rastier, 2001). Ainsi, l'auteur privilégie certaines propriétés lexicales, syntaxiques, sémantiques, structurelles pour répondre aux attentes de ses lecteurs. Le lecteur attend plusieurs indices pour reconnaître le genre (Biber et Conrad, 2009: 15), par exemple des formules d'introduction et de fin, une structure textuelle prédéfinie. Les propriétés communes à plusieurs genres discursifs définissent des champs génériques (catégories abstraites qui regroupent plusieurs genres ayant des caractéristiques communes), selon les définitions présentées dans l'introduction du numéro.

4 Par conséquent, nous étudions un faisceau des propriétés lexicales, morphosyntaxiques, syntaxiques, sémantiques et stylométriques (fréquences des signes de ponctuation, des mots longs et des mots courts) utiles pour la classification automatique par genre. Nous avons analysé un corpus comprenant plusieurs genres journalistiques, pour vérifier les hypothèses posées dans la littérature sur la caractérisation des genres discursifs du domaine journalistique (Ablali, 2012, Agnès, 2008, Grevisse, 2008, Revaz, 2001, Herman et Jufer, 2001, Lee, 1999). Sur la base de cette analyse, nous sélectionnons les propriétés qui s'avèrent les plus pertinentes pour la classification. Dans un premier temps, nous réalisons une classification de textes en sept genres discursifs du domaine journalistique (bulletin de circulation/météo, critique, éditorial, interview dialogué, nécrologie, recette de cuisine ${ }^{1}$, tribune libre), à l'aide de propriétés variées. Dans une deuxième étape, nous présentons des expériences destinées à classer les textes dans des catégories plus larges, selon le champ générique de l'opinion (éditorial, critique, tribune libre), le champ générique d'information (bulletin, recette de cuisine, nécrologie), l'interview représentant un champ à part, entre le champ de l'information et celui de l'opinion.

\section{Genre discursif et genre journalistique}

5 Dans cette section, nous introduisons les notions de genre discursif et de champ générique que nous avons adoptées pour la classification automatique dans le domaine du journalisme.

\subsection{Genre discursif et champ générique}

Le genre représente l'encrage social du texte et du discours (Adam, 1997 ; Rastier, 1989 : 37 ; Bakthine et Volochnikov, 1977). Du point de vue de Rastier (2001) ou de Biber et Conrad (2009), il est nécessaire d'interpréter les textes dans le contexte de production et de réception (pour un public visé, par un auteur spécifique). Dans cette perspective, le genre discursif représente un faisceau de normes communicationnelles qui varient selon le temps historique, le contexte social, le support médiatique. Ces conditions de production et de réception sont déterminantes pour la production et l'interprétation du texte aussi bien au niveau de l'expression (plan du texte, prosodie, formules d'introduction ou de fin...) et au niveau du contenu (thèmes, unités lexicales, ...). Ainsi, le genre possède un puissant caractère normatif imposant des contraintes au niveau 
lexical, syntaxique, sémantique et stylistique aux textes. Les contraintes se manifestent sous la forme d'indices variés dans la forme et le contenu représentés dans les textes. Ces contraintes ont un caractère fonctionnel précis (informer, divertir, argumenter) dans la situation de communication imposée (Biber et Conrad, 2009, Bouquet, 2004).

Nous adoptons ici la terminologie proposée dans l'article de Gérard (ce numéro) à propos de genres discursifs et de champs génériques. Les genres discursifs emploient des unités textuelles attendues par le récepteur du texte, regroupées par des règles de combinaison prédéfinies. Selon Biber et Conrad (2009), ces éléments sont identifiables par des indices de surface (fréquence des termes spécifiques, de certaines parties de discours, préférence pour des figures de style spécifiques etc.). Ces indices sont en partie repérables à l'aide des outils de TAL, ce que nous utilisons pour la classification automatique par genre.

D'autre part, selon Malrieu et Rastier (2001), un champ générique est un groupe de genres qui ont les mêmes objectifs fonctionnels et qui partagent un faisceau de propriétés communes. Par exemple, au sein des genres journalistiques, on distingue les champs génériques de l'information (compte rendu, fait divers, brève) et de l'opinion (tribune libre, éditorial, chronique). Ainsi, le champ générique de l'information vise à apporter des informations au public d'une manière objective. Il est caractérisé par un effacement complet du journaliste. Le champ générique de l'opinion nécessite une implication du journaliste ou de l'auteur de l'article dans le récit ou la présentation des faits pour convaincre le lecteur à partager ses opinions.

Dans le cadre de cette tâche de classification automatique, nous avons étudié des genres discursifs du domaine journalistique. Nous nous distinguons d'autres travaux visant la classification automatique qui détectent le domaine ou le thème (D'Hondt et al, 2013, Bechet et al, 2008). Nous étudions en particulier les propriétés communes des genres discursifs regroupés en champs génériques de l'information et de l'opinion dans le domaine journalistique.

\subsection{Les genres journalistiques}

10 Les genres journalistiques ont fait l'objet de nombreuses études en linguistique textuelle (Adam, 2001). Grosse (2000) propose une hiérarchie des genres journalistiques sur la base de l'évolution historique de ces genres discursifs. À l'ère du numérique, les genres journalistiques connaissent une évolution accélérée: les articles sont plus courts ou découpés en plusieurs parties (reliées par des hyperliens), les propos sont illustrés par des images ou par des articles reliés etc. Cette évolution impose des changements dans la perception des genres par les auteurs et les lecteurs de ces textes.

11 La fonction communicative (Martin-Lagardette, 1994) permet de classer les genres journalistiques en six catégories: l'information, l'opinion, le conseil, la fiction, le divertissement, et la publicité (Grosse et Seibold, 1996). Certains genres discursifs ont comme but la transmission des faits, dans le champ générique de l'information: portrait, nécrologie, reportage, fait divers, bulletin météorologique (Facques et Sanders, 2001). D'autres sont destinés à la transmission d'une opinion (faisant partie du champ générique de l'opinion) : analyse, éditorial, chronique, critique, tribune libre. L'éditorial ou la critique présentent l'opinion du journal ou du journaliste, alors que la tribune libre présente des opinions des personnes externes au journal. Pour servir à ces intentions communicationnelles, les manuels journalistiques préconisent certaines 
normes d'écriture (Mouriquand, 1997 ou Agnès, 2008) et des contraintes spécifiques à respecter dans la production d'un article appartenant à un genre discursif (Grevisse, 2008, Agnès, 2008). Les articles s'adressent au grand public, le style doit être concis et le vocabulaire non spécialisé.

La structure du texte est imposée pour certains genres : signature de l'article pour la tribune libre, les paragraphes marqués par un tiret et une succession de questions et de réponses pour l'interview dialoguée. Les guillemets sont très fréquents dans l'interview paraphrasée ou pour la revue de presse. Les dates ou les intervalles de temps sont souvent utilisés dans la nécrologie ou le portrait. La longueur de l'article peut représenter un facteur de distinction entre les divers genres. Les brèves sont des articles courts (même réduits à quelques lignes), alors que l'analyse ou le reportage sont plutôt des articles longs (une ou plusieurs colonnes ou pages).

Les caractéristiques lexicales, morphosyntaxiques, syntaxiques ou stylométriques sont aussi influencées par le public, mais aussi par le type d'information véhiculée (Lugrin, 2000). En effet, la critique est riche en vocabulaire de l'art, de la littérature ou du film, selon l'objet de l'article. La nécrologie privilégie le lexique spécifique de la mort (Revaz, 2001), alors que l'analyse ou l'éditorial utilise le vocabulaire du domaine politique ou économique. Le lexique d'opinion (verbes et adverbes d'opinion) est employé fréquemment dans les genres appartenant au champ générique de l'opinion: critique, tribune libre, éditorial. Les verbes de communication sont très fréquents dans les interviews dialoguées ou paraphrasés.

Certaines préférences pour des catégories lexicales spécifiques sont influencées par le genre discursif (Adam, 2001): l'éditorial privilégie les pronoms personnels de $1^{\text {ère }}$ personne du pluriel «nous» ou le pronom «on» qui permet d'effacer le sujet énonciateur (Lee, 1999) afin d'exprimer une opinion collective. Les interviews utilisent une alternance des pronoms personnels de $1^{\text {ère }}$ personne et de $2^{\mathrm{e}}$ personne puisqu'il s'agit du dialogue direct. Pour la critique ou la tribune libre, le pronom personnel de $1^{\text {ère }}$ personne («je» ou «nous») est utilisé car ces genres présentent l'opinion de l'auteur de l'article.

15 Pour notre étude, nous nous intéressons aux travaux identifiant les caractéristiques linguistiques et textuelles des genres discursifs que nous traitons. Ablali (2012) identifie plusieurs propriétés linguistiques spécifiques aux éditoriaux. Ainsi, la présence du pronom personnel « on » (qui inclut le lecteur), une fréquence supérieure des adjectifs et des noms par rapport aux verbes, et une prédilection pour le temps présent sont des propriétés utilisées pour convaincre le lecteur de la justesse des affirmations énoncées dans l'article. Les questions rhétoriques (marquées par le signe «?») suivies des réponses, sont fréquentes : elles permettent d'inclure le lecteur dans l'argumentation de l'éditorialiste (Lee, 1999, Herman et Jufer, 2001). Le signe «:» est utilisé pour énumérer plusieurs thèmes principaux de l'éditorial. Durrer (2001) indique que la tribune libre est caractérisée par des marques de l'énonciateur (pronom personnel " je », verbes de communication) et par un style soutenu, par rapport à d'autres genres du champ d'opinion, tels que les billets ou les chroniques.

Revaz (2001) identifie les caractéristiques linguistiques de la nécrologie, sur un corpus d'annonces signées par des journalistes ou par des dépêches d'agence. Elle met en évidence la prédilection pour le lexique de la mort (est mort, s'est éteint, a tiré sa révérence etc.) mais aussi pour certains procédés (apposition, anaphore nominale), comparaison et reformulation (surnommé $\mathrm{x}$ ). La présence des temps du passé (passé composé, plus- 
que-parfait) dépend de la taille de la nécrologie, si celle-ci se limite à une annonce de décès ou rappelle aussi les qualifications du défunt. Certaines structures syntaxiques typiques (Nom propre est mort/s'est éteint/a tiré sa révérence) sont construites à l'aide du lexique de la mort.

Ces études sont très peu exploitées dans le domaine de la classification automatique par genre.

\section{Classification automatique par genre}

Dans le domaine du traitement automatique des langues, le genre fait l'objet des nombreuses applications de classification automatique: extraction de terminologie (choix de textes spécialisés ou de vulgarisation) (Todiraşcu et Bertrand, 2014), identification automatique de néologismes (Todiraşcu, 2018) ou de sélection de textes adaptés aux dyslexiques (Gala et al., 2018).

Tout comme en linguistique textuelle, la classification automatique par genre utilise des acceptions différentes de cette notion, en fonction des applications dédiées. Le genre est désigné comme "un ensemble de documents qui partagent le même but communicatif » (Kessler et al, 1997). Les campagnes DEFT (Défi Fouille de Textes) s'intéressent régulièrement à la question de la classification automatique de documents par leur genre, le plus souvent en combinaison avec le domaine ou le sujet du document. Certains travaux s'intéressent à une classification par registre ${ }^{2}$ : Lecorvé et al. (2018), le discours académique vs. le discours de vulgarisation (Todiraşcu et Bertrand, 2014), ou encore des distinctions entre langue générale ou langue de spécialité (D'hondt et al, 2013, la campagne DEFT Bechet et al, 2008), la modalité (oral vs écrit) (Fang et Cao, 2010). D'autres travaux se situent au niveau du style : identification automatique de l'auteur (Stamatatos et al., 2009) ou du profil textuel (Dias et al., 2018). Plusieurs travaux sont dédiés aux genres spécifiques, tels que les genres journalistiques (Petrenz et Webber, 2011), les genres du Web (Mehler et al., 2010) ou les genres littéraires (Beauvisage, 2001).

Les systèmes de classification automatique des textes ont comme objectif de regrouper les documents par genre. Ces systèmes de classification représentent des documents sous forme d'ensemble de descripteurs (propriétés), qui sont utilisés pour construire le modèle de classification. Ce modèle est composé des descripteurs typiques pour chaque classe. On applique ce modèle aux textes non-annotés pour leur attribuer une classe identifiée automatiquement.

21 En fonction de la tâche de classification choisie, les descripteurs peuvent se situer au niveau lexical, syntaxique, morphosyntaxique ou sémantique. Des indicateurs stylométriques ou statistiques (calculés sur les textes bruts, tel que la longueur des phrases ou la fréquence des mots courts ou des mots longs, la fréquence des signes de ponctuation) ou la fréquence des mots pleins sont utilisés traditionnellement pour la classification par genre (Kessler et al., 1997), (Karlgren et Cutting, 1994). Les mots pleins dépendent $d u$ thème $d u$ document, par conséquent ces travaux réalisent plutôt une classification par thème que par genre.

La plupart du temps les propriétés utilisées pour la classification sont les catégories lexicales (noms, verbes, adjectifs, adverbes) (Malrieu et Rastier, 2001, Fang et Cao, 2010) ou des caractéristiques syntaxiques (fonctions syntaxiques, nombres de groupes 
nominaux complexes, types de sujets ou d'objets). Ces approches nécessitent un prétraitement des données (étiquetage morphosyntaxique, analyse syntaxique). Ces propriétés s'avèrent efficaces pour la classification des sous-genres journalistiques (Petrenz et Webber, 2011). Malgré quelques travaux qui s'appuient sur l'analyse de corpus et sur la notion de genre ou de registre (Lecorvé et al., 2018, Poudat et al., 2006, Todiraşcu et Bertrand, 2014), ou celle de style propre à l'auteur (Stamatatos et al., 2009) ou classement des profils utilisateurs (Dias et al., 2018). Les descripteurs appartenant au niveau lexical ou stylométrique sont privilégiés pour ces tâches de classification (Bechet et al., 2008).

Dans le cadre de cet article, nous défendons l'utilisation des propriétés motivées linguistiquement, identifiées à partir d'une analyse détaillée des genres discursifs journalistiques. Ainsi, nous explorons plusieurs catégories de propriétés (lexicales, morphosyntaxiques, stylométriques, syntaxiques) pour la classification par genre discursif dans un cadre spécifique, celui du Logoscope, plateforme de détection de candidats néologiques dans la presse.

\section{Le projet Logoscope}

Notre tâche de classification de documents appartenant aux genres journalistiques s'inscrit dans la continuité du projet Logoscope $e^{3}$ dont la plateforme en ligne répertorie les néologismes contemporains issus de la presse française, scrutée tous les jours depuis octobre 2014. Chaque document permet d'extraire une liste de mots inconnus (c'est-à-dire ne se trouvant pas dans les dictionnaires et lexiques existants). La plateforme repère des candidats néologiques crées par des procédés morphosyntaxiques ou syntaxiques, à l'aide des lemmes ou de l'analyse syntaxique. Ces candidats sont triés manuellement et stockés dans une base de données. Chaque néologisme validé fait l'objet d'une documentation qui indique le journal où il a été employé, sa date d'apparition, son contexte (plusieurs phrases), sa catégorie grammaticale, son procédé de création et trois thèmes associés (Gérard et al., 2014).

Malgré le fait que le Logoscope propose une documentation riche concernant le néologisme, notamment des thèmes, il ne détecte pas encore le genre du document dans lequel le néologisme a été créé la première fois, ou celui où il a été diffusé. Pourtant, le genre influence les procédés de création néologiques et les genres ne sont pas tous favorables à la création de néologismes (Gérard, et al., 2017, Gérard, 2011). Ainsi, il est difficile de trouver des néologismes dans des genres discursifs tels que les faits divers, les bulletins météo ou les recettes, genres qui ne sont pas néologènes (Gérard, 2018). La plupart des plateformes existantes pour la recherche de néologie, tel que Neoveille (Cartier, 2016), OBNEO (Cabré et al, 2003) s'intéressent aux procédés de construction des néologismes en contexte. OBNEO annote manuellement les genres du sous-corpus oral et les rubriques où le néologisme a été trouvé. Le genre de la page Web où le candidat est trouvé est documenté manuellement par Pompamo (Ollinger et Valette, 2010), dans sa base de données. L'identification du genre permet de compléter la documentation des néologismes, d'étudier la création et la diffusion des néologismes dans le contexte des genres différents.

Les expériences présentées dans cet article permettront à terme d'indiquer le genre du document dans lequel on trouve les néologismes proposés par la plateforme Logoscope. Ainsi, les textes accueillant les néologismes détectés pourront être classés 
automatiquement par leur champ générique (opinion, information) ainsi que par leur genre discursif (éditorial, bulletin météo, recette etc.). Pour réaliser cette classification automatique par champ générique et genre discursif, nous devons identifier les propriétés adaptées pour décrire les genres discursifs du domaine journalistique, sur la base des études des genres journalistiques (présentées dans la section 2.2) et de l'analyse de notre corpus (présentée en section 5.1).

\section{Méthodologie}

Nous avons adopté la définition de genre exposée par Biber et Conrad (2009). Le genre est décrit par une liste de paramètres situationnels (le public visé, l'auteur, les conditions de production et de réception du texte) et dépendant de la fonction visée par le texte (informatif, argumentatif, narratif, descriptif). Ces conditions imposent des choix stricts sur l'emploi du lexique, des figures de style ou des structures syntaxiques plus ou moins complexes. Pour mieux décrire les propriétés des genres journalistiques, nous avons identifié des études des propriétés des genres journalistiques (Ablali, 2012, Lee, 1999, Herman et Jufer, 2001, Revaz, 2001) et les particularités de ces genres, présentées dans les manuels de journalisme (Agnès, 2008, Grevisse, 2008, Grosse, 2000).

Partant de ces études des propriétés des genres journalistiques, nous proposons un modèle informatique pour la classification des champs génériques de l'opinion et de l'information. En effet, le champ de l'opinion est plus souvent porteur de néologismes (Gérard, 2018). Parmi les genres du champ de l'information nous avons retenu: le bulletin de circulation/météo, la nécrologie, la recette de cuisine. Les genres de l'opinion sont la critique, l'éditorial, la tribune libre. L'interview dialoguée se situe entre les deux champs, on trouve à la fois des opinions exprimées par les participants à l'entretien mais aussi des informations y sont véhiculées.

Pour établir les descripteurs les plus appropriés permettant de classer les champs génériques et les genres discursifs, nous avons procédé à une analyse de corpus de genres discursifs spécifiques (éditorial, nécrologie, tribune libre, etc.) et nous avons utilisé les recommandations des manuels de journalisme (Agnès, 2008, Grevisse, 2008). Nous avons complété la liste proposée par Biber et Conrad (2009), qui regroupe soixante-quatre propriétés morphosyntaxiques et syntaxiques (fréquence des noms, des adjectifs, de sous-classes d'adjectifs, des pronoms, des sujets et des objets pronominaux etc.) avec des propriétés proposées dans la littérature (vocabulaire spécifique de l'opinion, lexique de la nécrologie).

30 À partir de l'analyse de corpus, nous avons retenu cinquante-deux descripteurs et nous avons évalué l'influence des descripteurs dans la tâche de classification automatique par genre discursif et par champ générique, selon la méthodologie proposée par Todirașcu et Bertrand (2014), Todiraşcu (2018).

\subsection{Le corpus}

Pour nos expériences de classification, nous avons besoin d'un volume important de documents annotés en champs génériques et en genres discursifs. Les expériences réalisées sur un corpus annoté manuellement (Todiraşcu, 2018) par plusieurs annotateurs ont montré qu'il y a souvent des confusions entre les genres discursifs attribués aux documents. Malgré un nombre limité de catégories, les annotateurs 
peuvent proposer une annotation erronée ou des catégories différentes pour le même document.

Nous avons extrait des articles journalistiques de la base FACTIVA ${ }^{4}$, contenant des articles de la presse francophone et étrangère, disponible en ligne. FACTIVA permet l'accès à la presse quotidienne nationale et régionale (Le Monde, Libération, Le Figaro, The New York Times, etc.), aux revues professionnelles et spécialisées (Alternatives économiques, Challenges, etc.), aux dépêches d'agences de presse ou aux sites Web d'actualité. Cette base propose une annotation en genre discursif du domaine journalistique.

33 Nous avons retenu sept genres discursifs (bulletin de circulation/météo, critique, éditorial, interview dialoguée, nécrologie, recette de cuisine, tribune libre). Les genres sont regroupés en deux champs génériques : opinion (éditorial, critique, tribune libre), information (bulletin, nécrologie, recette de cuisine). L'interview dialoguée se situe entre les deux champs génériques de l'opinion et de l'information. Nous avons choisi entre 100 et 164 textes par genre discursif, les textes sont généralement courts (Tableau 1).

Ce corpus est tokenisé, lemmatisé, étiqueté avec la catégorie lexicale et annoté en dépendances avec l'analyseur syntaxique MATE (Bohnet, 2009). Ces propriétés sont à la fois utiles pour l'analyse manuelle de corpus et pour l'extraction automatique de propriétés à l'aide des patrons lexico-syntaxiques (Todiraşcu et Bertrand, 2014).

\begin{tabular}{|l|l|l|l|}
\hline Genre discursif & Nb de documents & Nb d'unités lexicales et de signes de ponctuation & Nb de types \\
\hline Bulletin & 164 & 73374 & 8170 \\
\hline Critique & 103 & 26760 & 6276 \\
\hline Editorial & 100 & 35701 & 7087 \\
\hline Interview & 138 & 211098 & 17886 \\
\hline Nécrologie & 133 & 9262 & 1932 \\
\hline Récette & 112 & 13327 & 7516 \\
\hline Tribune libre & 120 & 40870 & \\
\hline
\end{tabular}

Tableau 1 : La composition du corpus extrait de FACTIVA.

Pour vérifier les hypothèses présentées dans la littérature et dans les manuels de journalisme, nous avons procédé à une analyse détaillée de corpus.

\subsection{Analyse de corpus}

Nous avons évalué les propriétés stylométriques, lexicales, morphosyntaxiques et syntaxiques de chaque genre, afin d'identifier des propriétés typiques par analyse contrastive. Ainsi, nous avons analysé le corpus à l'aide du concordancier Antconc 
(Anthony, 2009) pour comparer les différences de propriétés des genres discursifs étudiés et pour vérifier les hypothèses avancées dans la littérature.

Afin de comparer les propriétés des divers genres, nous utilisons la fréquence relative d'une propriété. Ce paramètre est calculé selon la formule suivante, où FT représente la fréquence absolue de la propriété (le nombre total d'occurrences de la propriété) et NbTokens est le nombre total de tokens (unités lexicales et signes de ponctuation) du texte :

\section{$F R=\frac{F T}{\text { Nbtokens }}$}

Dans notre étude, nous avons considéré que les propriétés dont la fréquence est supérieure que la moyenne sont considérées comme spécifiques pour le genre discursif étudié. Dans toutes les sections suivantes, nous avons utilisé la fréquence relative des propriétés pour comparer les genres.

Les propriétés stylométriques et lexicales ont été analysées à l'aide du concordancier Antconc, les autres ont été calculées à l'aide des patrons lexico-syntaxiques proposés par (Todiraşcu et Bertrand, 2014). Dans les sections suivantes, nous détaillons les différences observées entre les deux champs génériques et les genres discursifs sélectionnés.

\subsubsection{Les propriétés stylométriques}

Les propriétés stylométriques sélectionnées sont des propriétés utilisées aussi dans la littérature (Bechet et al., 2008) : la longueur moyenne des textes, la longueur moyenne des phrases, la fréquence des mots très courts (moins de 3-4 lettres), de longueur moyenne (entre 5 et 8 lettres) ou très longs (plus de 9 lettres), la fréquence de signes de ponctuation (signes d'interrogation, d'exclamation, le point virgule, le double point). Ces propriétés sont efficaces dans plusieurs tâches de classification par genre (Kessler et al, 1997).

\begin{tabular}{|l|l|l|l|l|l|}
\hline & $\begin{array}{l}\text { Longueur moyenne du } \\
\text { texte }\end{array}$ & $\begin{array}{l}\text { Signe } \\
\text { d'interrogation }\end{array}$ & $\begin{array}{l}\text { Signe } \\
\text { d'exclamation }\end{array}$ & Guillemets & $\begin{array}{l}\text { Mots } \\
\text { longs }\end{array}$ \\
\hline Bulletin & 388,89 & 754,77 & 1044,34 & $\mathbf{1 0 1 7 2 , 5 2}$ & 106666,54 \\
\hline Interview & $\mathbf{1 6 8 5 , 8 0}$ & $\mathbf{8 3 8 0 1 , 5 0}$ & $\mathbf{6 8 7 6 , 3 8}$ & 1525,46 & $\mathbf{1 2 4 1 1 0 , 8 5}$ \\
\hline Nécrologie & 80,62 & 0,00 & 939,85 & 0,00 & 112011,49 \\
\hline Recette & 146,16 & 1116,07 & $\mathbf{4 6 3 8 , 5 5}$ & 94,48 & 73340,10 \\
\hline Tribune & $\mathbf{6 8 7 , 6 2}$ & 5352,78 & 3774,99 & 169,29 & $\mathbf{1 4 4 8 1 4 , 4 1}$ \\
\hline Critique & 299,12 & 6749,72 & 2947,98 & $\mathbf{2 1 9 6 , 8 2}$ & 113721,07 \\
\hline
\end{tabular}




\begin{tabular}{|l|l|l|l|l|l|}
\hline Editorial & 453,42 & 13493,35 & 5686,64 & 413,75 & 130622,60 \\
\hline
\end{tabular}

Tableau 2 : Les propriétés stylométriques les plus significatives avec la fréquence relative (FR) : en gras les valeurs supérieures à la moyenne

41 Dans notre corpus, la longueur moyenne des textes s'avère significative pour distinguer les genres de l'opinion (tribune libre, éditorial) ${ }^{5}$ des autres genres de l'information. En effet, la longueur moyenne des textes des genres d'information présents dans le corpus est inférieure à la moyenne globale calculée pour ce paramètre. L'interview se détache par la longueur beacoup plus importante que les autres genres. La critique est plus courte que d'autres genres d'opinion, car il s'agit souvent de critique de films ou de livres. Le signe d'interrogation est typique pour l'interview (une succession des questions et de réponses), suivi par l'éditorial qui pose des questions rhétoriques. Cette propriété caractérise aussi la tribune et la critique, genres discursifs d'opinion. Les signes d'interrogation représentent alors un bon indice pour distinguer les genres d'information et d'opinion. Ce constat est aussi valable pour le signe d'exclamation, à l'exception notable de la recette qui appartient au champ générique de l'information. Les guillemets sont typiques pour les bulletins (car souvent on reprend des propos d'autres dépêches) et pour la critique (reprise de répliques ou des citations du texte original). Ce descripteur permet la distinction d'un type particulier de genre (critique, nécrologie). Les mots longs sont spécifiques pour la tribune, l'éditorial, l'interview et la critique. Il s'agit des genres d'opinion, ayant une structure du texte plus complexe.

\subsubsection{Les propriétés morphosyntaxiques}

Dans cette catégorie de descripteurs, entreront les catégories lexicales et les souscatégories lexicales, les temps, les modes et la diathèse des verbes. Nous avons regardé la fréquence de chaque catégorie lexicale, des sous-catégories (pronoms personnels, démonstratifs, possessifs etc.) dans les genres discursifs étudiés. Les résultats sont présentés dans le tableau 3 :

\begin{tabular}{|l|l|l|l|l|l|}
\hline & $\begin{array}{l}\text { Nom } \\
\text { propre }\end{array}$ & Adverbe & Conj. & Part. présent & Adjectifs \\
\hline Bulletin & $\mathbf{5 5 8 9 3 , 8 6}$ & $\mathbf{4 5 1 8 0 , 6 1}$ & 423,28 & $\mathbf{3 4 1 5 , 4 7}$ & 9817,15 \\
\hline Interview & 45771,08 & $\mathbf{5 7 3 4 1 , 5 3}$ & $\mathbf{6 6 5 , 2 2}$ & 2638,78 & $\mathbf{1 8 1 7 8 , 4 4}$ \\
\hline Nécrologie & $\mathbf{1 0 7 2 2 9 , 9 9}$ & 19106,52 & 0,00 & 1472,47 & $\mathbf{1 4 9 1 7 , 4 8}$ \\
\hline Recette & 23489,19 & 16600,75 & 456,41 & 2221,23 & 6284,76 \\
\hline Tribune & $\mathbf{5 6 9 0 3 , 7 6}$ & $\mathbf{4 5 1 2 0 , 8 3}$ & $\mathbf{7 1 1 , 1 4}$ & $\mathbf{4 4 8 7 , 4 0}$ & $\mathbf{2 3 2 6 9 , 7 7}$ \\
\hline Critique & $\mathbf{8 3 5 9 9 , 7 6}$ & 32546,36 & $\mathbf{7 9 1 , 4 4}$ & $\mathbf{4 2 8 2 , 8 4}$ & 13640,23 \\
\hline
\end{tabular}




\begin{tabular}{|l|l|l|l|l|l|}
\hline Editorial & 50813,00 & 56812,24 & $\mathbf{7 7 0 , 6 5}$ & $\mathbf{4 5 0 7 , 0 8}$ & $\mathbf{2 0 5 3 0 , 3 9}$ \\
\hline
\end{tabular}

Tableau 3 : Les fréquences relatives de plusieurs catégories lexicales et propriétés morphosyntaxiques (FR) critique (les auteurs de film, d'œuvres littéraires), la tribune (souvent signée par des
auteurs identifiés) et le bulletin (riche en noms propres de lieu). Les adverbes sont plus nombreux dans l'éditorial ou dans l'interview : on a souvent des précisions sur le temps, l'espace, la manière ou des jugements de valeur. La conjonction, témoin d'une structure phrastique complexe, est plus fréquente pour les genres d'opinion (la critique, l'éditorial et la tribune) que pour les genres d'information. Les adjectifs sont aussi typiques pour les textes longs (tribune, éditorial, interview) et plus souvent utilisés dans le champ générique de l'opinion. Le participe présent modifiant des groupes nominaux apparait fréquemment dans l'éditorial, la tribune ou encore la critique.

Les pronoms relatifs introduisent des subordonnées relatives et sont caractéristiques pour le champ générique de l'opinion et pour l'interview (tableau 4). Les pronoms personnels de $1^{\text {ère }}$ personne sont typiques pour l'interview (une personne répond aux questions de la deuxième personne), la critique (qui exprime l'opinion de l'auteur sur l'objet de la critique) ou la tribune libre. Plus surprenant, nous retrouvons une fréquence importante dans les bulletins, alors que dans l'éditorial, la fréquence est moindre. Dans ces derniers, l'éditorialiste préfère l'emploi du pronom de pluralité «nous » ou encore « on », pour exprimer le point de vue du journal, et non pas du seul journaliste qui a écrit le texte.

Tous ces paramètres indiquent une structure de phrase complexe, plus élaborée, contenant des modifieurs adverbiaux ou adjectivaux, introduits par un participe présent ou des subordonnées relatives, qui semblaient spécifiques aux genres d'opinion.

\begin{tabular}{|l|l|l|l|l|}
\hline & Pronom Relatif & On & Pron. pers. je & Nous \\
\hline Bulletin & 6322,80 & 265,39 & $\mathbf{1 6 1 , 1 9}$ & 169,06 \\
\hline Interview & $\mathbf{1 3 2 5 3 , 1 3}$ & $\mathbf{7 9 7 , 4 9}$ & $\mathbf{5 7 7 9 , 5 1}$ & $\mathbf{1 3 4 7 , 7 9}$ \\
\hline Nécrologie & 5758,27 & 51,87 & 0,00 & $\mathbf{3 7 5 , 2 0}$ \\
\hline Recette & 1490,64 & 110,82 & 0,00 & 0,00 \\
\hline Tribune & $\mathbf{1 2 4 3 5 , 4 2}$ & $\mathbf{3 9 8 , 4 2}$ & $\mathbf{6 0 7 , 7 0}$ & $\mathbf{3 3 2 , 3 6}$ \\
\hline Critique & $\mathbf{1 0 4 9 0 , 2 9}$ & $\mathbf{3 9 8 , 4 2}$ & $\mathbf{1 2 6 0 , 4 1}$ & 254,14 \\
\hline Editorial & $\mathbf{1 3 2 1 0 , 0 0}$ & $\mathbf{5 9 0 , 5 0}$ & 83,64 & $\mathbf{2 9 5 , 5 0}$ \\
\hline
\end{tabular}

Tableau 4 : La fréquence relative des pronoms par genre 
46

" générique «on» est plus souvent employé dans l'interview, suivi par l'éditorial (inclusion de l'auteur dans l'ensemble de l'équipe du journal), la critique et la tribune. Ce pronom est utilisé plus fréquemment dans le champ générique de l'opinion que dans celui de l'information. La forme « nous » est très utilisée par l'interview, mais beaucoup moins dans les autres genres de l'opinion.

\subsubsection{Les propriétés syntaxiques}

Nous avons étudié plusieurs structures syntaxiques et les fonctions syntaxiques associées. Le type de sujet est en particulier intéressant: complexe, pronominal, pronominal inversé. Le sujet complexe (subordonnée, groupe nominal complexe) est plus fréquent dans les genres du champ de l'opinion, dont une des caractéristiques est une structure de phrase plus élaborée : la tribune, la critique et l'éditorial.

pronominal est typique pour l'interview et pour l'éditorial, même s'il ne s'agit pas des mêmes pronoms (pour l'interview les pronoms de $1^{\text {ère }}$ et $2^{\mathrm{e}}$ personnes, pour l'éditorial les pronoms «nous » ou « on »). En revanche, la séquence de noms propres désignant une personne ou une œuvre sont typiques pour la nécrologie et pour la critique. Enfin, la tournure impersonnelle est employée très fréquemment en nécrologie. En ce qui concerne le passif, il est très fréquent dans les bulletins mais aussi dans les genres du champ de l'opinion (critique, tribune, éditorial) ou dans l'interview, il est difficile de juger de la pertinence pour la classification.

\begin{tabular}{|l|l|l|l|l|l|l|}
\hline & $\begin{array}{l}\text { Sujet } \\
\text { complexe }\end{array}$ & $\begin{array}{l}\text { Sujet } \\
\text { pronominal }\end{array}$ & Passif & $\begin{array}{l}\text { Séquence de } \\
\text { noms } \\
\text { propres }\end{array}$ & $\begin{array}{l}\text { Sujet } \\
\text { pronominal } \\
\text { inversé }\end{array}$ & $\begin{array}{l}\text { Tournures } \\
\text { impersonnelles }\end{array}$ \\
\hline Bulletin & 437099,81 & 218502,28 & $\mathbf{2 1 1 7 , 8 8}$ & 5848,68 & 218502,28 & 554,88 \\
\hline Interview & 346249,79 & $\mathbf{4 8 1 6 4 2 , 2 5}$ & 1387,64 & 8432,43 & $\mathbf{4 8 1 6 4 2 , 2 5}$ & 1336,58 \\
\hline nécrologie & 357089,15 & 325787,68 & 973,55 & $\mathbf{2 2 7 7 8 , 9 3}$ & 325787,68 & $\mathbf{4 6 3 7 , 7 8}$ \\
\hline recette & 309077,38 & 186656,75 & 53,15 & 1197,31 & 186656,75 & 597,63 \\
\hline tribune & $\mathbf{4 9 6 9 1 4 , 3 0}$ & 273202,69 & 1411,80 & 11913,39 & 273202,69 & 1228,19 \\
\hline critique & 488104,34 & 293706,24 & 2012,11 & 21146,65 & 293706,24 & 1736,82 \\
\hline éditorial & 466044,49 & 316813,78 & 1320,07 & 10303,69 & 316813,78 & 1870,80 \\
\hline
\end{tabular}

Tableau 5 : La fréquence relative de quelques propriétés syntaxiques

Pour le calcul de ces fréquences, nous avons utilisé AntConc appliqué sur le corpus annoté syntaxiquement (sujet). Pour d'autres propriétés, tels que les séquences de noms propres, les tournures impersonnelles, les appositions ont été calculées à l'aide des patrons lexico-syntaxiques proposés par Todirascu et Bertrand (2014) que nous avons mentionnés dans la section 5.2. 


\subsubsection{Les propriétés sémantiques} quelques classes de propriétés sémantiques : verbes de communication, verbes de croyance, d'opinion, le lexique de la mort et de la nécrologie, lexique de noms de métiers et de professions. Chaque classe a été utilisée pour un genre particulier ou un champ générique. Ainsi, le vocabulaire de la mort est typique pour la nécrologie. Les noms de professions vont apparaître dans la nécrologie ou dans l'interview (si celui-ci évoque le passé de la personne). Les champs génériques de l'opinion (éditorial, critique, tribune libre) privilégient les prises de position, présentent une prise de position de la part de l'auteur de l'article ou de celui qui est l'acteur de l'entretien. Cette prise de position est exprimée à la première personne (je, nous), par des verbes de communication (dire, parler, expliquer, informer, etc.) par des verbes exprimant une opinion (penser, regretter, croire) ou des adjectifs portant un jugement (bon, excellent, très bon, mauvais, difficile, etc.). Les marqueurs argumentatifs (mais, cependant, etc.) sont aussi utilisés pour identifier les genres d'opinion alors que les verbes de conseil identifient le genre d'information.

\begin{tabular}{|l|l|l|l|l|l|}
\hline & $\begin{array}{l}\text { Verbes } \\
\text { d'opinion }\end{array}$ & Verbes de conseil & $\begin{array}{l}\text { Adjectifs } \\
\text { d'opinion }\end{array}$ & Marqueurs & $\begin{array}{l}\text { Verbes de } \\
\text { communication }\end{array}$ \\
\hline Bulletin & 928,17 & $\mathbf{2 1 6 5 , 5 8}$ & $\mathbf{1 0 0 9 , 3 4}$ & 0,00 & 3051,42 \\
\hline Interview & $\mathbf{1 8 2 5 , 5 8}$ & 542,41 & 798,49 & $\mathbf{7 1 , 0 2}$ & 3185,47 \\
\hline Nécrologie & $\mathbf{1 9 7 3 , 4 4}$ & $\mathbf{1 0 2 7 , 6 6}$ & 288,80 & 0,00 & 2640,29 \\
\hline Recette & 522,75 & 279,57 & $\mathbf{9 6 7 2 , 7 4}$ & 0,00 & $\mathbf{8 2 2 2 , 5 4}$ \\
\hline Tribune & $\mathbf{2 6 5 3 , 4 0}$ & 389,98 & 691,14 & $\mathbf{5 1 , 0 9}$ & 2734,69 \\
\hline Critique & 1822,77 & $\mathbf{1 7 3 4 , 4 7}$ & 479,39 & $\mathbf{3 2 , 3 6}$ & 2345,72 \\
\hline Editorial & $\mathbf{3 2 0 8 , 3 9}$ & 319,47 & 681,94 & $\mathbf{7 3 , 8 9}$ & 3283,07 \\
\hline
\end{tabular}

Tableau 6 : Les propriétés sémantiques les plus saillantes (et leur fréquence relative FR) pour caractériser les genres discursifs

51 En vue d'une classification automatique, nous avons créé des lexiques pour chaque catégorie sémantique. Les marqueurs discursifs sont les plus efficaces quant à la distinction entre genres de l'information (où l'absence de ces marqueurs est saillante) et de l'opinion. Nous constatons que les verbes d'opinion sont très fréquents dans les éditoriaux, les tribunes libres (champ générique de l'opinion) et les interviews. Plus surprenant, la nécrologie est riche en verbes d'opinion ce qui semble contredire l'hypothèse que les verbes d'opinion sont spécifiques aux genres d'opinion. Certains verbes sont ambigus ce qui explique la présence élevée de cette catégorie de verbes dans la nécrologie.

La faible fréquence de verbes de communication contredit les hypothèses que nous avons formulées : on s'attend à les retrouver d'une manière abondante dans les genres 
d'opinion. Un degré important d'ambiguïté les caractérise : dans certains contextes ils n'ont pas de fonction communicative mais d'explicitation, d'information, d'explication. Dans le calcul de propriétés, nous ne pouvons pas prendre en compte le contexte, il n'est alors pas possible de désambiguïser les occurrences de celles utilisées dans d'autres objectifs.

\section{Premiers résultats de classification automatique}

L'étude du corpus a confirmé des observations réalisées sur un autre corpus annoté manuellement et sur les genres communs (Todiraşcu, 2018). Les propriétés morphosyntaxiques sont parmi les plus saillantes pour certains genres, ce qui confirme les résultats obtenus par Petrenz et Webber (2011), Malrieu et Rastier (2001), Poudat et al. (2006). Nous avons identifié une préférence marquée pour les pronoms personnels de $1^{\text {ère }}$ personne dans la critique et l'interview. Nous constatons que les préférences pour les pronoms impersonnels et les pronoms de la $2^{\mathrm{e}}$ personne pluriel (vous), pour les phrases interrogatives et exclamatives sont très marquées dans les éditoriaux, ce qui confirme les observations réalisées dans la littérature (Ablali, 2012). Dans le champ générique de l'information, il y a une préférence pour les noms propres (nécrologie, bulletin). Cette propriété apparait aussi dans le champ de l'opinion (notamment dans la critique, où l'objet de la critique est un auteur, un film, un livre etc.).

Partant de l'analyse de corpus, nous avons proposé une extraction des propriétés de niveaux variés, parfois le simple calcul de la fréquence sur le corpus étiqueté (selon la formule présentée dans la section Méthodologie) ou des patrons d'extraction plus complexes. Nous avons d'abord réalisé des expériences de classification avec les sept genres discursifs (en gardant entre 100 et 160 textes par genre), à l'aide de la plateforme Weka (Hall et al, 2009). Nous avons utilisé les 52 propriétés étudiées à l'aide de Antconc. Nous avons évalué plusieurs algorithmes de classification supervisés: réseaux bayesiennes, arbres de décision (J48), machines à vecteurs support (SVM). Le plus performant s'avère l'algorithme SVM, pour lequel nous présentons les résultats. Pour cet algorithme, nous avons obtenu une exactitude de 81,41 \% (le nombre d'instances correctement classées). Certains genres présentent des difficultés de classification : il y a beaucoup de confusions entre la tribune, la critique et l'éditorial (voir la matrice de confusion dans le tableau 7). La matrice de confusion indique le nombre d'instances correctement classées et le nombre d'instances mal classées (entre critiques et tribunes, entre éditoriaux et tribunes). Sur la diagonale du tableau 7, nous identifions le nombre de documents bien classés.

\begin{tabular}{|l|l|l|l|l|l|l|l|}
\hline Bulletin & Interview & nécrologie & recette & tribune & critique & éditorial & classe \\
\hline 108 & 1 & 4 & 0 & 1 & 3 & 1 & Bulletin \\
\hline 0 & 132 & 0 & 0 & 6 & 0 & 0 & Interview \\
\hline 1 & 0 & 126 & 0 & 0 & 4 & 2 & nécrologie \\
\hline 0 & 0 & 2 & 109 & 0 & 0 & 1 & recette \\
\hline 1 & 1 & 7 & 0 & 51 & 13 & 20 & tribune \\
\hline
\end{tabular}




\begin{tabular}{|l|l|l|l|l|l|l|l|}
\hline 11 & 2 & 4 & 0 & 12 & 63 & 11 & critique \\
\hline 2 & 1 & 1 & 0 & 25 & 8 & 49 & éditorial \\
\hline
\end{tabular}

Tableau 7 : La matrice de confusion obtenue par classification avec l'algorithme SVM (un des plus populaires pour la classification automatique des documents) par validation croisée $(n=10)$

Pour l'algorithme SVM, nous obtenons la meilleure F-mesure calculée selon la formule suivante :

$$
F=\frac{2 \times P \times R}{P+R}
$$

où $\mathrm{P}$ est la précision (le nombre de documents correctement classés pour chaque genre/ le nombre de documents attribués au genre) et le rappel (le nombre de documents correctement classés pour chaque genre/ le nombre total de documents correctement attribués au genre).

\begin{tabular}{|l|l|l|l|}
\hline Précision & Rappel & F-mesure & Classe \\
\hline 0,878 & 0,915 & 0,896 & Bulletin \\
\hline 0,964 & 0,957 & 0,96 & Interview \\
\hline 0,875 & 0,947 & 0,91 & nécrologie \\
\hline 1 & 0,973 & 0,986 & recette \\
\hline 0,537 & 0,548 & 0,543 & tribune \\
\hline 0,692 & 0,612 & 0,649 & critique \\
\hline 0,583 & 0,57 & 0,576 & éditorial \\
\hline
\end{tabular}

Tableau 8 : La précision, le rappel, la F-mesure obtenues pour les 7 genres discursifs

En terme de F-mesure, le système obtient les meilleurs résultats pour la classe des recettes ou de l'interview, alors que les résultats les plus faibles sont obtenus pour l'éditorial, la tribune et la critique (il y a beaucoup de confusions entre ces trois genres discursifs).

Nous avons procédé à plusieurs expériences par types de propriétés. Pour chaque expérience, nous avons sélectionné les propriétés stylométriques, lexicales, syntaxiques, sémantiques. Toutefois, pour chaque catégorie de propriétés considérées seules, l'exactitude est très faible (autour de $60 \%$ au lieu de $81 \%$ obtenue pour l'ensemble de propriétés). Ainsi, pour notre corpus et les genres choisis, un faisceau de propriétés de plusieurs catégories permet d'obtenir les meilleurs résultats. Parmi les propriétés stylométriques, nous constatons que la longueur moyenne des phrases, les mots très longs (plus de 9 lettres), les signes d'interrogation, la présence des nombres, et la longueur du texte sont les plus efficaces. En ce qui concerne la catégorie lexicale, 
les propriétés sélectionnées sont la fréquence des substantifs, des verbes, des adverbes, des pronoms relatifs, des adjectifs relationnels. Les verbes d'opinion et les sujets pronominaux et simples (groupes nominaux sans modifieurs) sont également retenus.

Enfin, nous avons choisi de restreindre les expériences aux champs génériques de l'information (bulletin, recette, nécrologie) et d'opinion (critique, tribune libre, éditorial). L'interview est un genre discursif qui peut présenter les opinions de la personne qui participe à l'interview mais aussi informe sur certains sujets. Nous avons regroupé les documents dans trois classes différentes, deux qui correspondent aux champs génériques de l'information et de l'opinion et celle de l'interview (qui est à part). Avec ces trois classes et les propriétés mentionnées, nous obtenons une exactitude de $91,31 \%$, avec les meilleurs résultats pour l'interview (certains indices tels que les signes d'interrogation et les pronoms sont très caractéristiques) :

\begin{tabular}{|l|l|l|l|}
\hline & Précision & Rappel & F-mesure \\
\hline information & 0,909 & 0,934 & 0,921 \\
\hline interview & $\mathbf{0 , 9 6 3}$ & $\mathbf{0 , 9 4 9}$ & $\mathbf{0 , 9 5 6}$ \\
\hline opinion & 0,894 & 0,869 & 0,881 \\
\hline
\end{tabular}

Tableau 9 : La précision, le rappel et la F-mesure obtenues avec l'algorithme SVM

Nous pouvons aussi constater qu'il y a confusion entre le champ générique de l'opinion, de l'information et les interviews.

\section{Conclusion et perspectives}

61 Les expériences réalisées dans le cadre de cet article montrent qu'il est possible de faire une annotation automatique préliminaire en champ générique de l'information ou de l'opinion. L'exactitude de $91 \%$ est améliorée par rapport aux résultats obtenus avec les sept genres discursifs (exactitude de $81 \%$ ). La difficulté principale consiste en l'absence de critères linguistiques détaillés et calculables automatiquement permettant de trancher entre tribune, critique et éditorial. Les propriétés les plus pertinentes appartiennent à tous les niveaux : lexical, morphosyntaxique, syntaxique, sémantique, stylométrique et cette observation est valable également pour d'autres corpus (Todiraşcu, 2018, Todiraşcu et Bertrand, 2014). Les propriétés dépendantes du thème (vocabulaire spécifique) sont peu nombreuses (sauf pour la nécrologie). Les résultats de l'analyse de corpus ont validé partiellement des hypothèses proposées dans la littérature. La classification entre opinion, information et interview est déjà une information importante pour la documentation des candidats néologiques proposés par le Logoscope.

62 Nos observations doivent être complétées sur un volume de données plus important extrait de Factiva, et doivent être étendues à d'autres genres du domaine journalistique. Certaines propriétés sont corrélées (longueur du texte et complexité des phrases) et on doit identifier ces corrélations pour garder uniquement la propriété la 
plus efficace. D'autre part, les autres genres peuvent aussi poser des problèmes de classification s'ils ont des propriétés trop similaires (comme pour les genres d'opinion).

\section{BIBLIOGRAPHIE}

ABLALI, D., 2012, « La cooccurrence auto-constituante sur un corpus de presse. », Communication présentée à l'occasion du Colloque de Cerisy « Documents, Textes, CEuvres », Cérisy, consultable sur http://www.revue-texto.net/.

ADAM, J.-M., 1997, « Genres, textes, discours : pour une reconception linguistique du concept de genre ", Revue belge de philologie et d'histoire, $\mathrm{n}^{\circ}$ 75, p. 665-681.

ADAM, J.-M., 2001, « Genres de la presse écrite et analyse de discours », Semen [En ligne], 13 | 2001, mis en ligne le 30 avril 2007, consulté le 31 juillet 2018. URL : http:// journals.openedition.org/semen/2597 AGNÈS, Y., 2008, Manuel de journalisme, Paris, La Découverte.

ANTHONY, L., 2009, « Issues in the design and development of software tools for corpus studies: The case for collaboration », in P. Baker (éd.), Contemporary corpus linguistics, London, Continuum Press, p. 87-104.

BAKTHINE, M. et VOLOCHINOV, V. N., 1977, Le marxisme et la philosophie du langage, Paris, Éditions de Minuit.

BEAUVISAGE, T., 2001, « Morphosyntaxe et genres textuels. Exploiter des données morphosyntaxiques pour l'étude statistique des genres textuels : application au roman policier. ", Traitement Automatique des Langues, $\mathrm{n}^{\circ}$ 42, p. 579-608.

BECHET, F., El-Bèze M. et Torres-Moreno J.-M., 2008, Actes du quatrième défi fouille de texte, DEFT2008, Avignon, France, p. 27-36.

BIBER, D. et CONRAD, S., 2009, Register, Genre and Style, Cambridge, Cambridge University Press. BOHNET, B., 2009, « Efficient Parsing of Syntactic and Semantic Dependency Structures. », in Proceedings of Conference on Natural Language Learning (CoNLL), p. 67-72.

BOUQUET, S., 2004, « Linguistique générale et linguistique des genres (Introduction au numéro) », Langages, 2004/1 ( $\left.n^{\circ} 153\right)$, p. 3-14.

CABRÉ, M.-T. et alii, 2003, « L'observatoire de néologie : conception, méthodologie, résultats et nouveaux travaux. », dans Jean-François Sablayrolles (éd.), L'innovation Lexicale, Paris, Honoré Champion, p. 125-147.

D’HONDT, E. et alii, 2013, « Text Representations for Patent Classification », Computational Linguistics, $\mathrm{n}^{\circ} 39$, p. 755-775.

DIAS, C.-E., GAINON de FORSAN de GABRIAC, C., GUIGUE, V. et GALLINARI, P., 2018, « RNN \& modèle d'attention pour l'apprentissage de profils textuels personnalisés », CORIA-TALN 2018. 
DURRER, S., 2001, « De quelques affinités génériques du billet », Semen [En ligne], 13 | 2001, mis en ligne le 04 mai 2012, consulté le 31 juillet 2018. URL : http://journals.openedition.org/semen/ 2600.

FACQUES, B. et SANDERS, C., 2004, « Textes journalistiques et analyse contrastive du genre en didactique. ", Langages, $\mathrm{n}^{\circ} 153$, p. 86-97.

FANG, A.C, CAO, J., 2010, « Enhanced Genre Classification through Linguistically Fine-Grained POS Tags », PACLING Proceedings, 2010.

GALA, N., FRANÇOIS, T., JAVOUREY-DREVET, L., ZIEGLER, J.-C., 2018, « Vers une simplification automatique de textes pour une meilleure compréhension », Langue Française « L'apprentissage de la lecture en français langue maternelle et seconde ", Armand Colin (sous presse).

GÉRARD, C., 2011, « Création lexicale, sens et textualité. », PhiN. Philologie im Netz, n 56, p. 1-30.

GÉRARD, C., 2018, « Variabilité du langage et productivité lexicale. Problèmes et propositions méthodologiques ", Neologica, $n^{\circ} 12$, Lexique : nouveauté et productivité, p. 23-45.

GÉRARD, C., ce volume, « Linguistique des genres : objet et méthode. Statut culturel des genres et variétés génériques », Linx 78 .

GÉRARD, C., FALK, I. et BERNHARD, D., 2014, « Traitement automatisé de la néologie : pourquoi et comment intégrer l'analyse thématique? ", $4^{e}$ Congrès Mondial de Linguistique Française, $\mathrm{n}^{\circ} 8, \mathrm{p}$. 2627-2646.

GÉRARD, C. et LACOSTE, C., 2014, « La création lexicale dans les écrits de combattants de la Première Guerre mondiale », La Première Guerre mondiale et la langue, colloque international, Paris, France.

GÉRARD, C. et alii, 2017, « Le Logoscope : observatoire des innovations lexicales en français contemporain ", dans Joaquín García Palacios et alii (éds), La neologia en las lenguas romanicas : recursos, estrategias y nuevas orientaciones, Bern, Peter Lang.

GREVISSE, B., 2008, Écritures journalistiques. Stratégies rédactionnelles, multimédia et journalisme narratif, Bruxelles, De Boeck, collection «Info-Com».

GROSSE, E.U., 2000, « Évolution et typologie des genres journalistiques. Essai d'une vue d'ensemble », Semen, $n^{\circ} 13$, p. 15-36.

GROSSE, E.U. et SEIBOLD, E., 1996, Panorama de la presse parisienne, Frankfort/Berlin, Peter Lang.

HALL, M. et alii, 2009, " The WEKA Data Mining Software: An Update », SIGKDD Exploration Newsletter, $\mathrm{n}^{\circ} 11$, p. 10-18.

HALLIDAY, M.A.K, 1978, Language as a Semiotic System, London, Edward Arnold

HERMAN, T. et JUFER, N., 2012, « L'éditorial, « vitrine idéologique du journal » ? », Semen [En ligne], 13 | 2001, mis en ligne le 04 mai 2012, consulté le 31 juillet 2018

KARLGREN, J. and CUTTING, D., 1994, "Recognizing Text Genres with Simple Metrics Using Discriminant Analysis.", Proceedings of COLING 94, p. 1071-1075.

KESSLER, B., NUNBERG, G. and SCHÜLTZE, H., 1997, "Automatic detection of text genre.", Proceedings of EACL'97, p. 32-38.

LECORVÉ, G., AYATS, H., FOURNIER, B., MEKKI, J., CHEVELU, J., BATTISTELLI, D., BÉCHET, N., 2018, « Construction conjointe d'un corpus et d'un classifieur pour les registres de langue en français », CORIA-TALN 2018. 
LEE, N.-S., 1999, Caractérisation et reconnaissance des genres. Propositions didactiques. Le cas de l'éditorial dans la presse écrite, Thèse de Sciences du Langage de l'Université de Rouen.

LUGRIN, G., 2000, «Critères de typologisation des genres de la presse écrite ", Études de Lettres, $\mathrm{n}^{\circ}$ 3, p. 13-72.

MALRIEU, D., et RASTIER, F., 2001, « Genres et variations morphosyntaxiques », Traitements automatiques du langage, $\mathrm{n}^{\circ} 42$, p. 547-577.

MARTIN-LAGARDETTE, J.-L., 1994, Guide de l'écriture journalistique. Écrire, informer, convaincre, Paris, Syros.

MEHLER, A., SHAROFF, S., SANTINI, M. (eds), 2010, Genres on the web: Computational Models and Empirical Studies. Springer Series: Text, Speech and Language Technology (Series Editors:Ide, Nancy, Véronis, Jean).

MOURIQUAND, J., 1997, L'écriture journalistique, Paris, PUF, collection « Que-Sais-Je ?».

OLLINGER, S., et VALETTE, M., 2010, «La créativité lexicale : des pratiques sociales aux textes », dans Maria Teresa Cabré et alii (éds), Actes del I Congrés Internacional de Neologia de les llengües romaniques (CINEO'08), Barcelona, 07-10 maig 2008, Barcelona, Publicacions de l'Institut Universitari de Lingüística Aplicada (IULA) de la Universitat Pompeu Fabra (UPF), p. 25-40.

PETRENZ, P. et WEBBER, B., 2011, « Stable Classification of Text Genres. », Computational Linguistics, $\mathrm{n}^{\circ}$ 37, p. 385-393.

POUDAT, C., CLEUZIOU, G., et CLAVIER, V., 2006, « Catégorisation de textes en domaines et genres : complémentarité des indexations », Document numérique, $\mathrm{n}^{\circ}$ 9, p. 61-76.

RASTIER, F., 1989, Sens et textualité, Paris, Hachette.

RASTIER, F., CAVAZZA, M., et ABEILLE, A., 1994, Sémantique pour l'analyse. De la linguistique à l'informatique, Paris : Masson.

RASTIER, F. 2001, Arts et sciences du texte, PUF, Paris.

REVAZ, F., 2001, « La nécrologie : un genre rédactionnel ? », Semen [En ligne], 13.

STAMATATOS, E., FAKOTAKIS, N., KOKKINAKIS, G., 2001, Computers and the Humanities, Volume 35, Issue,2 Pages 193-214, Kluwer Academic Publishers.

TODIRAŞCU, A., et BERTRAND, G., 2014, « ClassYN : classer les documents selon le genre textuel », dans Mejri Salah et alii (éds), Actes des Journées scientifiques du réseau LTT, Paris, Éditions des archives contemporaines, p. 520-530.

TODIRAŞCU, A., 2018, « Néologie et genres textuels : comment caractériser les genres journalistiques pour la classification automatique? », dans D. Bernhard, M. Boisseau, C. Gérard, T. Grass, A. Todirascu (eds.) La néologie en contexte : Cultures, situations, textes, Limoges : LambertLucas, p. 281-297.

STAMATATOS, E., FAKOTAKIS, N., et KOKKINAKIS G., 2000, « Text Genre Detection Using Common Word Frequencies. » Proceedings of the 18th International Conference on Computational Linguistics (COLING2000).

\section{NOTES}

1. Cette catégorie est présente dans les catégories de la base Factiva et dans des presses spécialisées. 
2. Registres de langues (soutenu, familier, courant).

3. Le Logoscope est un projet Idex financé par l'Université de Strasbourg (2012-2015) et par la DGFLF (depuis 2015) (responsables scientifiques Delphine Bernhard et Christophe Gérard) : http://logoscope.unistra.fr/

4. https://www.dowjones.com/products/factiva

5. Cette propriété est variable pour les médias disponibles en ligne, d'une page à un paragraphe et même d'un journal à un autre. Cependant, c'est une propriété saillante pour les textes qui font partie de notre corpus.

\section{RÉSUMÉS}

La classification automatique par genre est une tâche difficile, pour les systèmes de Traitement automatique des langues, due à la diversité des définitions du genre et à l'absence d'une définition adaptée pour une approche automatique. Le genre discursif détermine les procédés d'écriture et de réception du texte (Rastier, 1989, Bouquet, 2004). En effet, par son caractère normatif, le genre influence les choix de vocabulaire, de syntaxe, de style (Biber et Conrad, 2009), y compris les procédés de création lexicale. Nous exploitons ces propriétés linguistiques pour la classification automatique des genres discursifs, appartenant aux champs génériques de l'opinion et de l'information dans le domaine du journalisme. Les propriétés choisies pour la classification automatique sont établies après une analyse de corpus de genres discursifs et champs génériques journalistiques.

The automatic genre classification represents a difficult task for NLP systems, due to the absence of a unique definition of genre, useful for automatic classification. The discursive genre is determinant for text production and interpretation (Rastier, 1989, Bouquet, 2004). The genre influences the choice of lexical words, syntactic structures or styles (Biber et Conrad, 2009) or the process of neologism creation. We exploit the linguistic properties induced by the genre to automatically classify newspapers discourse genre or more generic categories (information vs opinion). The properties are established on the basis of a linguistic analysis of the newspapers genres and categories.

\section{INDEX}

Mots-clés : Classification automatique par genre, genres journalistiques, propriétés statistiques, morphosyntaxiques, syntaxiques pour la classification

Keywords : Automatic classification by genre, journalistic genres, statistical, morpho-syntactic, syntactic properties for classification

\section{AUTEUR}

\section{AMALIA TODIRASCU}

FDT, LiLPa, Université de Strasbourg 